\title{
NOTES ON SMOOTH FUNCTION GERMS ON VARIETIES
}

\author{
SHYŪICHI IZUMIYA AND SACHIKO MATSUOKA
}

\begin{abstract}
We study smooth function germs on varieties from the point of view of Thom-Mather theory.
\end{abstract}

0. Introduction. In this paper we obtain an algebraic criterion for $k$-determinacy (in a certain sense) of smooth function germs on a variety relative to the group of germs of diffeomorphisms preserving the variety. It is a generalization of the algebraic criteria for $k$-determinacy of smooth function germs at the origin and on manifolds with boundary $[\mathbf{4}, \mathbf{5}]$. On the other hand, all function germs which are classified by Arnol'd and Mather in $[\mathbf{1}, \mathbf{5}]$ have only isolated singularities at the origin. We consider the possibility of studying smooth function germs with nonisolated singularities.

All results in this paper are also valid in the complex analytic category.

The authors would like to thank the referee for careful readings.

1. $R_{I(h)}$-equivalence. In this section we introduce the crucial idea for studying function germs on varieties. Let $\mathcal{E}_{n}$ be the ring of germs at 0 of smooth function germs $f:\left(\mathbf{R}^{n}, 0\right) \rightarrow \mathbf{R}$ and $\mathfrak{M}_{n}$ be the unique maximal ideal in $\mathcal{E}_{n}$. Let $\mathfrak{M}(n, p)$ be the set of germs at 0 of a smooth map $h:\left(\mathbf{R}^{n}, 0\right) \rightarrow\left(\mathbf{R}^{p}, 0\right)$. For any $h \in$ $\mathfrak{M}(n, p)$, we define the ring homomorphism $h^{*}: \mathcal{E}_{p} \rightarrow \mathcal{E}_{n}$ by $h^{*}(f)=f \circ h$. We fix $h \in \mathfrak{M}(n, p)$. By $R_{I(h)}$ we denote the group of diffeomorphism germs $\phi:\left(\mathbf{R}^{n}, 0\right) \rightarrow$ $\left(\mathbf{R}^{n}, 0\right)$ such that $\phi^{*}(I(h))=I(h)$, where $I(h)=h^{*}\left(\mathfrak{M}_{p}\right) \mathcal{E}_{n}$.

DEFINITION 1.1. For any fixed $h \in \mathfrak{M}(n, p)$, two function germs $f, g \in \mathfrak{M}_{n}$ are

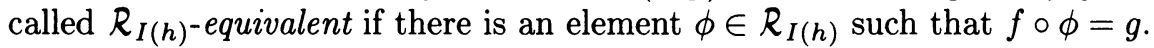

REMARK. If $\phi \in R_{I(h)}$, then $\phi\left(h^{-1}(0)\right)=h^{-1}(0)$. Hence, we may have $R_{I(h)^{-}}$ equivalence of function germs as right equivalence of function germs on the variety $h^{-1}(0)$.

Since we do not know whether the set of $k$-jets of elements of $R_{I(h)}$ is a Lie group or not, we consider the following notion: Let $\mathcal{K}(n, p)$ be the group of $\mathcal{K}$-equivalence in the sense of Mather [6].

DEFinition 1.2. Two pairs of map germs $\left(f_{1}, h_{1}\right),\left(f_{2}, h_{2}\right) \in \mathfrak{M}_{n} \times \mathfrak{M}(n, p)$ are called $(R, \mathcal{K})$-equivalent if there exists $(\phi, \Phi) \in \mathcal{K}(n, p)$ such that $\left(f_{1} \circ \phi, h_{1} \circ \phi\right)=$ $\left(f_{2}, \Phi \circ\left(\mathrm{id}, h_{2}\right)\right)$.

After we had submitted this paper, the referee pointed out that this idea had also been developed in $[\mathbf{3}]$.

Received by the editors May 8, 1985.

1980 Mathematics Subject Classification. Primary 58C27; Secondary 32B30.

Key words and phrases. Smooth function germs, variety, finite determinancy, nonisolated singularities. 
By Proposition 2.3 in [6], we have the following lemma:

LemMa 1.3. Suppose $h \in \mathfrak{M}(n, p)$ and $f, g \in \mathfrak{M}_{n}$. Then the following are equivalent:

(i) $f, g$ are $R_{I(h)}$-equivalent.

(ii) $(f, h)$ and $(g, h)$ are $(R, \mathcal{K})$-equivalent.

By this lemma, the $(R, K)$-equivalence theory can be used to study the $R_{I(h)^{-}}$ equivalence theory.

For any $(f, h) \in \mathfrak{M}_{n} \times \mathfrak{M}(n, p)$, let $\theta(f)$ (respectively $\left.\theta(h)\right)$ be the $\mathcal{E}_{n}$-module of $C^{\infty}$-vector field germs along $f$ (respectively $h$ ) at the origin. We put $\theta(n)=$ $\theta\left(\operatorname{id}_{\mathbf{R}^{n}}\right)$. Then we define an $\mathcal{E}_{n^{-}}$-homomorphism $t(f, h): \theta(n) \rightarrow \theta(f) \oplus \theta(h)$ by $t(f, h)(\xi)=d f \circ \xi \oplus d h \circ \xi$. By analogy with the $\mathcal{K}$-tangent space of map germ we define the $(R, \mathcal{K})$-tangent space for $(f, h) \in \mathfrak{M}_{n} \times \mathfrak{M}(n, p)$ by

$$
T(R, \mathcal{K})(f, h)=t(f, h)\left(\mathfrak{M}_{n} \theta(n)\right)+0 \oplus h^{*}\left(\mathfrak{M}_{p}\right) \theta(h) .
$$

We now define $\iota: \theta(f) \rightarrow \theta(f) \oplus \theta(h)$ by $\iota(\eta)=\eta \oplus 0$. By an easy calculation, we have the following lemma:

LEMMA 1.4. For any $(f, h) \in \mathfrak{M}_{n} \times \mathfrak{M}(n, p), \iota^{-1}(T(R, \mathcal{K})(f, h))=\{t f(\xi) \mid \xi \in$ $\left.\mathfrak{M}_{n} \theta(n), \operatorname{th}(\xi) \in h^{*}\left(\mathfrak{M}_{p}\right) \theta(h)\right\}$.

Because of the above observation, we define an ideal in $\mathcal{E}_{n}$ as follows: For any $(f, h) \in \mathfrak{M}_{n} \times \mathfrak{M}(n, p)$, we put

$$
\begin{aligned}
J_{I(h)}(f)=\left\{\xi_{1} \frac{\partial f}{\partial x_{1}}+\cdots+\xi_{n} \frac{\partial f}{\partial x_{n}} \mid \xi_{i}\right. & \in \mathfrak{M}_{n}, \\
& \left.\xi_{1} \frac{\partial h}{\partial x_{1}}+\cdots+\xi_{n} \frac{\partial h}{\partial x_{n}} \in h^{*}\left(\mathfrak{M}_{p}\right) \mathcal{E}(n, p)\right\}
\end{aligned}
$$

where $\mathcal{E}(n, p)=\mathcal{E}_{n} \times \cdots \times \mathcal{E}_{n}$ (product of $p$-copies of $\left.\mathcal{E}_{n}\right)$ and $\partial h / \partial x_{i}=\left(\partial h_{1} / \partial x_{i}\right.$, $\left.\ldots, \partial h_{p} / \partial x_{i}\right)$ for the coordinate representation $h=\left(h_{1}, \ldots, h_{p}\right)$.

We may consider the ideal $J_{I(h)}(f)$ as the space of infinitesimal variation of $f$ with respect to $R_{I(h)}$-equivalence.

2. $I$-finite determinacy. Let $I$ be an ideal in $\mathcal{E}_{n}$.

DEFINITION 2.1. Two function germs $f, g \in I$ are called $I$ - $k$-jet equivalent if $f-g \in \mathfrak{M}_{n}^{k} I$. This relation is an equivalence relation and we denote by $j_{I}^{k} f$ the equivalence class represented by $f \in I$. We call $j_{I}^{k} f$ the $I$ - $k$-jet of $f$.

We remark that if $I=\mathfrak{M}_{n}$ the $I$ - $k$-jet of $f$ is the ordinary $k$-jet of $f$.

DEFINITION 2.2. Suppose $h \in \mathfrak{M}(n, p)$. The function germ $f \in I$ is called $I$ $k$-determined relative to $R_{I(h)}$ if $f$ and $g$ are $R_{I(h)}$-equivalent for any $g \in I$ with $j_{I}^{k} g=j_{I}^{k} f$. The function germ $f \in I$ is called $I$-finitely determined relative to $R_{I(h)}$ if $f$ is $I$ - $k$-determined relative to $R_{I(h)}$ for some $k$.

We remark that the $I-k$-determinacy is the $M$-determinacy in [7] in the case where $M=\mathfrak{M}_{n}^{k} I$. As the order of the $I$-finite determinacy we have the following proposition:

Proposition 2.3. Let $I$ be a finitely generated ideal in $\mathcal{E}_{n}$. Suppose $h \in$ $\mathfrak{M}(n, p)$ and $f \in I^{2}$. If

$$
\mathfrak{M}_{n}^{k-1} I \subset J_{I(h)}(f)+\mathfrak{M}_{n}^{k} I,
$$

then $f$ is $I^{2}$-k-determined relative to $R_{I(h)}$. 
The proof of Proposition 2.3 is the same as that of the following proposition which gives a more strict estimate in the case where $I=I(h)$. If

Proposition 2.4. Suppose that $I=I(h)$ for some $h \in \mathfrak{M}(n, p)$. Let $f \in I^{2}$.

$$
\mathfrak{M}_{n}^{k-1} I^{2} \subset J_{I(h)}(f)+\mathfrak{M}_{n}^{k} I^{2}
$$

then $f$ is $I^{2}$-k-determined relative to $R_{I(h)}$.

PROOF. The proof is almost the same as that of Proposition 1 in [5]. Suppose that $g \in I^{2}$ has the same $I^{2}$ - $k$-jet as $f$. We define $F(x, t)=f(x)+t(g(x)-f(x))$. We consider $F$ as an element of $\varepsilon_{n+1}$, the ring of germs at $\left(0, t_{0}\right)$. We denote its maximal ideal by $\mathfrak{M}_{n+1}$. We have inclusions $\mathcal{E}_{n} \subset \mathcal{E}_{n+1}$ and $\mathfrak{M}_{n} \subset \mathfrak{M}_{n+1}$. Let

$$
\begin{aligned}
J^{*}(F)=\left\langle\left\{\xi_{1} \frac{\partial F}{\partial x_{1}}+\cdots+\xi_{n} \frac{\partial F}{\partial x_{n}} \mid\right.\right. & \xi_{i} \in \mathfrak{M}_{n}, \\
& \left.\left.\xi_{1} \frac{\partial h}{\partial x_{1}}+\cdots+\xi_{n} \frac{\partial h}{\partial x_{n}} \in h^{*}\left(\mathfrak{M}_{p}\right) \mathcal{E}(n, p)\right\}\right\rangle_{\varepsilon_{n+1}} .
\end{aligned}
$$

By the condition

$$
\xi_{1} \frac{\partial h}{\partial x_{1}}+\cdots+\xi_{n} \frac{\partial h}{\partial x_{n}} \in h^{*}\left(\mathfrak{M}_{p}\right) \mathcal{E}(n, p),
$$

we remark that

$$
J_{I(h)}(f) \subset J^{*}(F)+\mathfrak{M}_{n}^{k} I^{2} \mathcal{E}_{n+1} .
$$

By Nakayama's lemma, $\mathfrak{M}_{n}^{k-1} I^{2} \varepsilon_{n+1} \subset J^{*}(F)$.

The only difference of the remainder of the proof is to check that the one parameter family of local diffeomorphisms preserves the ideal $I(h)$. But this is ensured by the condition that

$$
\xi_{1} \frac{\partial h}{\partial x_{1}}+\cdots+\xi_{n} \frac{\partial h}{\partial x_{n}} \in h^{*}\left(\mathfrak{M}_{p}\right) \mathcal{E}(n, p) . \quad \text { Q.E.D. }
$$

A necessary condition for $I$ - $k$-determinacy is given as follows:

PROPOSITION 2.5. Let I be a finitely generated ideal in $\mathcal{E}_{n}$ whose generators are real analytic function germs. Suppose that $h \in \mathfrak{M}(n, p)$ and $f \in I$ are also real analytic germs.

If $f$ is $I$ - $k$-determined relative to $R_{I(h)}$, then

$$
\mathfrak{M}_{n}^{k} I \subset J_{I(h)}(f) .
$$

ProOF. The condition $(*)$ is equivalent to the following condition:

$$
\mathfrak{M}_{n}^{k} I \theta(f) \oplus 0 \subset T(R, \mathcal{K})(f, h) .
$$

Let $\mathcal{K}(n, p)^{l}$ be the Lie group of $l$-jets of elements in $\mathcal{K}(n, p)$. There is a $\mathcal{K}(n, p)^{l}$ action on $J^{l}(n, 1) \oplus J^{l}(n, p)$ induced by $(R, \mathcal{K})$-equivalence. We denote by $J^{l}\left(f+\mathfrak{M}_{n}^{k} I\right)$ the set of $l$-jets of elements in $f+\mathfrak{M}_{n}^{k} I$. Since $J^{l}\left(f+\mathfrak{M}_{n}^{k} I\right)$ is an affine subspace of $J^{l}(n, 1) \oplus J^{l}(n, p)$, its tangent space at $j_{0}^{l} f$ is

$$
J^{l}\left(\mathfrak{M}_{n}^{k} I\right)=\left(\mathfrak{M}_{n}^{k} I \theta(f) \oplus 0+\mathfrak{M}_{n}^{l+1} \theta(f) \oplus \mathfrak{M}_{n}^{l+1} \theta(h)\right) /\left(\mathfrak{M}_{n}^{l+1} \theta(f) \oplus \mathfrak{M}_{n}^{l+1} \theta(h)\right) .
$$


Since $f$ is $I$ - $k$-determined relative to $R_{I(h)}$, we have

$$
\begin{aligned}
\frac{\mathfrak{M}_{n}^{l+1} I \theta(f) \oplus 0+\mathfrak{M}_{n}^{l+1} \theta(f) \oplus \mathfrak{M}_{n}^{l+1} \theta(h)}{\mathfrak{M}_{n}^{l+1} \theta(f) \oplus \mathfrak{M}_{n}^{l+1} \theta(h)} \\
\subset \frac{T(R, K)(f, h)+\mathfrak{M}_{n}^{l+1} \theta(f) \oplus \mathfrak{M}_{n}^{l+1} \theta(h)}{\mathfrak{M}_{n}^{l+1} \theta(f) \oplus \mathfrak{M}_{n}^{l+1} \theta(h)}
\end{aligned}
$$

for any $l \geq k$. If we take the projective limit of the above inclusion as $l \rightarrow \infty$, then we have the following inclusion:

$$
\mathfrak{M}_{n}^{k} I \theta(f) \oplus 0 \subset T(R, \mathcal{K})(f, h)+\mathfrak{M}_{n}^{\infty} \theta(f) \oplus \mathfrak{M}_{n}^{\infty} \theta(h) .
$$

This inclusion means that the condition $(* *)$ is true in the category of formal power series. But, since the generators of $I, h$ and $f$ are all real analytic germs, we have the condition $(* *)$ in the real analytic category by the theorem in [2]. Q.E.D.

If we take $I=\mathfrak{M}_{n}$ and $h\left(x_{1}, \ldots, x_{n}\right)=x_{1}$, we have the following corollary:

COROLlaRY 2.6 (JANECZKO [4]). Let $f \in \mathfrak{M}_{n}$.

(a) If

$$
\mathfrak{M}_{n}^{k} \subset\left\langle x_{1} \frac{\partial f}{\partial x_{1}}\right\rangle_{\mathcal{E}_{n}}+\mathfrak{M}_{n}\left\langle\frac{\partial f}{\partial x_{2}}, \ldots, \frac{\partial f}{\partial x_{n}}\right\rangle_{\mathcal{E}_{n}}+\mathfrak{M}_{n}^{k+1},
$$

then $f$ is $k$-determined relative to $G_{L}$.

(b) If $f$ is $k$-determined relative to $G_{L}$, then

$$
\mathfrak{M}_{n}^{k+1} \subset\left\langle x_{1} \frac{\partial f}{\partial x_{1}}\right\rangle_{\mathcal{E}_{n}}+\mathfrak{M}_{n}\left\langle\frac{\partial f}{\partial x_{2}}, \ldots, \frac{\partial f}{\partial x_{n}}\right\rangle_{\mathcal{E}_{n}} .
$$

Here, $G_{L}$ is the group of diffeomorphism germs $\phi$ such that $\phi(L)=L$ where $L=$ $\left\{x_{1}=0\right\}$.

If we take $I=\left\langle x_{2}, \cdots, x_{n}\right\rangle_{\mathcal{E}_{n}}$ and $h\left(x_{1}, \ldots, x_{n}\right)=\left(x_{2}, \ldots, x_{n}\right)$, we have the following corollary:

COROLlaRY 2.7 (SIERSMA [8]). Let $f \in I^{2}$ be a real analytic function germ.

(a) If $\mathfrak{M}_{n}^{k-2} I^{2} \subset \tau(f)+\mathfrak{M}_{n}^{k-1} I^{2}$, then $f$ is $k$-determined in the sense of Siersma.

(b) If $f$ is $k$-determined in the sense of Siersma, then $\mathfrak{M}_{n}^{k-1} I^{2} \subset \tau(f)$. Here.

$$
\tau(f)=\mathfrak{M}_{n}\left\langle\frac{\partial f}{\partial x_{1}}\right\rangle_{\mathcal{E}_{n}}+\left\langle x_{2}, \ldots, x_{n}\right\rangle_{\mathcal{E}_{n}}\left\langle\frac{\partial f}{\partial x_{2}}, \ldots, \frac{\partial f}{\partial x_{n}}\right\rangle_{\mathcal{E}_{n}} .
$$

3. Smooth function germs with nonisolated singularities. Let $I$ be an ideal in $\mathcal{E}_{n}$. For any $f \in \mathfrak{M}_{n}$, we define $J(f)=\left\langle\partial f / \partial x_{1}, \ldots, \partial f / \partial x_{n}\right\rangle \mathcal{E}_{n}$. If $f \in I^{2}$ then $J(f) \subset I$. We denote the zero point set of $I$ by $V(I)$. Let $\Sigma(f)$ be the set of singular points of $f$. It is equal to $V(J(f))$. Hence, if $f \in I^{2}$ then $\Sigma(f) \supset V(I)$. By the propositions in the previous section, we have the following proposition:

PROPOSITION 3.1. Let I be a finitely generated ideal in $\mathcal{E}_{n}$ whose generators are all real analytic germs. Suppose that $h \in \mathfrak{M}(n, p)$ and $f \in I^{2}$ are real analytic germs. If $f$ is $I^{2}$-finitely determined relative to $R_{I(h)}$, then $V(I)=\Sigma(f)$.

Proof. Since $f \in I^{2}$, we have $\Sigma(f) \supset V(I)$. By Proposition 2.5, there exists a nonnegative integer $k$ such that $\mathfrak{M}_{n}^{k} I^{2} \subset J_{I(h)}(f) \subset J(f)$. Hence, we have $V(I)=V\left(I^{2}\right)=V\left(\mathfrak{M}_{n}^{k} I^{2}\right)=V(J(f))=\Sigma(f)$. Q.E.D. 
EXAMPLE 3.2. Let $h:\left(\mathbf{R}^{2}, 0\right) \rightarrow\left(\mathbf{R}^{2}, 0\right)$ be a map germ given by $h(x, y)=(x, y)$. Put $I=\left\langle x^{3}-y^{2}\right\rangle \mathcal{E}_{n}$. Consider the function germ $f(x, y)=\left(x^{3}-y^{2}\right)^{2} \in I^{2}$. Then

$$
J_{I(h)}(f)=\mathfrak{M}_{2} J(f)=\left\langle x^{3}\left(x^{3}-y^{2}\right), x y\left(x^{3}-y^{2}\right), y^{2}\left(x^{3}-y^{2}\right)\right\rangle_{\varepsilon_{2}} .
$$

Since $x^{3}\left(x^{3}-y^{2}\right), x^{2} y\left(x^{3}-y^{2}\right), x y^{2}\left(x^{3}-y^{2}\right), y^{3}\left(x^{3}-y^{2}\right)$ generate $\mathfrak{M}_{2}^{3} I$, by Proposition $2.3, f$ is $I^{2}$-4-determined relative to right equivalence.

EXAMPLE 3.3. Let $h:\left(\mathbf{R}^{2}, 0\right) \rightarrow(\mathbf{R}, 0)$ be a function germ given by $h(x, y)=$ $x^{2}-y^{2}$. Put $I=I(h)=\left\langle x^{2}-y^{2}\right\rangle \mathcal{E}_{2}$.

(1) Consider the function germ $f_{1}(x, y)=\left(x^{2}-y^{2}\right)^{2} \in I^{2}$. Then

$$
\begin{aligned}
& J_{I(h)}\left(f_{1}\right)=\left\{\xi(x, y) 4 x\left(x^{2}-y^{2}\right)-\eta(x, y) 4 y\left(x^{2}-y^{2}\right) \mid\right. \\
& \left.\xi(x, y), \eta(x, y) \in \mathfrak{M}_{2}, \xi(x, y) 2 x-\eta(x, y) 2 y \in\left\langle x^{2}-y^{2}\right\rangle_{\mathcal{E}_{2}}\right\} .
\end{aligned}
$$

If we let $\xi(x, y)=\frac{1}{4} x$ and $\eta(x, y)=\frac{1}{4} y$, then $\left(x^{2}-y^{2}\right)^{2} \in J_{I(h)}\left(f_{1}\right)$. Since $\left(x^{2}-y^{2}\right)^{2}$ generates $\mathfrak{M}_{2}^{0} I^{2}=I^{2}$, by Proposition 2.4, $f_{1}$ is $I^{2}$-1-determined relative to $R_{I(h)}$.

(2) Consider the function germs $f_{2}(x, y)=x\left(x^{2}-y^{2}\right)^{2}$ and $f_{3}(x, y)=y\left(x^{2}-y^{2}\right)^{2}$. By the same calculation as in (1), $f_{2}$ and $f_{3}$ are $I^{2}$-2-determined relative to $R_{I(h)}$.

Since $\Sigma\left(f_{i}\right)=V\left(x^{2}-y^{2}\right)(i=1,2,3), f_{1}, f_{2}$ and $f_{3}$ are all nonfinitely determined function germs in the usual sense (cf. [5]). However, $\exp (x)\left(x^{2}-y^{2}\right)^{2}$ and $\left(x^{2}-y^{2}\right)^{2}$ (respectively $\sin (x)\left(x^{2}-y^{2}\right)^{2}$ and $\left.x\left(x^{2}-y^{2}\right)^{2}\right)$ are right equivalent. This is an assertion which does not follow from the previous results on determinacy.

\section{REFERENCES}

1. V. I. Arnol'd, Singularity theory, London Math. Soc. Lecture Note Ser., vol. 53, Cambridge Univ. Press, Cambridge and New York, 1981.

2. M. Artin, On the solution of analytic equations, Invent. Math. 5 (1968), 277-291.

3. A. Dimca, Function germs on isolated hypersurface singularities, Compositio Math. 53 (1984), 245-268.

4. S. Janeczko, On algebraic criteria for $k$-determinacy of germs of smooth functions on manifolds with boundary, Demonstratio Math. 4 (1982), 1113-1123.

5. J. Mather, unpublished note on right equivalence.

6. _ Stability of $C^{\infty}$-mappings. III: Finitely determined map germs, Inst. Hautes Études Sci. Publ. Math. 35 (1969), 127-156.

7. A. A. du Plessis, On the determinacy of smooth map germs, Invent. Math. 58 (1980), 107-160.

8. D. Siersma, Isolated line singularities, Proc. Sympos. Pure Math., vol. 40, part 2, Amer. Math. Soc., Providence, R. I., 1983, pp. 485-496.

Department of Mathematics, FaCulty of Science, HokKaido University, SAPPORO 060, JAPAN

Department of MAThematics, NARA Women's University, NARA 630, Japan 